\title{
LAS RELACIONES DE CAUSALIDAD ENTRE EL GASTO PÚBLICO Y EL PRODUCTO EN MÉXICO: ¿EXISTE EVIDENCIA DE CAMBIO ESTRUCTURAL?
}

\author{
Luis Galindo*
}

Facultad de Economía

Universidad Nacional Autónoma de México

Rolando Cordera

Facultad de Economía

Universidad Nacional Autónoma de México

(Recibido 30 de septiembre 2005, aceptado 6 de diciembre 2005)

\section{Resumen}

El objetivo principal del presente trabajo es evaluar la, ley de Wagner y la hipótesis Keynesiana en un contexto de análisis multivariado usando datos anuales de la economía mexicana para el períado 1970-2004. Los resultados indican la existencia de una relación de cointegración entre el ingreso per-capita, la inversión y el gasto público. Los valores de los coeficientes del vector de cointegración, sugieren que la inversión y el gasto público tienen un impacto positivo en el PIB per capita. Sin embargo, ambas variables reportan un coeficiente menor a uno, lo cual rechaza la hipótesis de Wagner. Las pruebas de exogeneidad débil indican que el ingreso per-capita y el gasto público son variables endógenas. En tanto las pruebas de no-causalidad en el sentido de Granger muestran que no existe hay causalidad entre ambas variables. En general el análisis realizado, confirma un efecto positivo de la inversión y el gasto público en el PIB per capita, pero se rechaza el cumplimento de los casos extremos de la ley de Wagner y la hipótesis keynesiana.

\section{Abstract}

The main objective of this study is to evaluate Wagner's law and the Keynesian paradigm in a multivariate context using Mexican annual data for the period 1970-2004. The main results indicate the existence of a cointegrating relationship between per-capita income, investment and public expenditure. The values of the coefficients suggest that investment and public expenditure have a positive impact on income. However, both variables have coefficients less than one rejecting Wagner's law. The weak exogeneity tests indicate that per-capita income and public expenditure are endogenous; meanwhile the Granger non-causality tests do not support the bypothesis of public expenditure causing per-capita income. Also, percapita income changes dot no cause public expenditure. In general the analysis confirms the

* Posgrado en Economía, Oficinas administrativas no. 2, primer piso, Av. Universidad 3000, Cd. Universitaria, UNAM., C. P. 04510, Deleg. Coyoacán, México, D.F., México. Teléfono +52(55) 5622-2341 ext. 41, 43,85. Correo electrónico: gapaliza@servidor.unam.mx

Agradecemos los comentarios de Leonardo Lomelí y Horacio Catalán, así como el apoyo en las estimaciones econométricas de Jaime Olivares. Desde Luego se aplica el descargo usual de los errores. Este trabajo fue financiado por el proyecto PAPIIT-UNAM IN-304702. 
positive effect of investment and public expenditure on income but rejects the two extreme cases of the Wagner law and the Keynesian paradigm.

Clasificación JEL: C20, H50

Palabras clave: Keynes, Wagner, Cointegración y Causalidad

\section{Introducción}

La capacidad de la política fiscal para contribuir al crecimiento económico es un tema que ha estado en el centro del debate de la política económica en México en al menos las tres últimas décadas. En este contexto, existe un marcado interés por identificar las relaciones de causalidad entre el gasto público y el producto y la posible presencia de posibles cambios estructurales en estas relaciones. En la literatura económica reciente existen a este respecto dos posiciones extremas para explicar la relación entre estas dos variables. Por un lado, la tradición Keynesiana de la demanda efectiva postula que el gasto público tiene un efecto positivo en el ingreso y que, por tanto, el gasto público induce o es un motor del crecimiento económico (Keynes, 1936). Por el otro lado, la hipótesis de Wagner (Wagner, 1890 y Wagner y Weber, 1977) considera que el crecimiento económico genera un aumento del gasto público y que por tanto, en este caso, la causalidad va del producto al gasto público.

La literatura en torno a este debate, para el caso de México, muestra también un importante grado de intensidad. De hecho, esta discusión en México esta marcado por el debate en torno al tamaño del estado y el cambio estructural en la relación entre el producto y el gasto público. Esto es, existe la hipótesis de que la función del gobierno de contribuir al crecimiento económico se modificó en las últimas dos décadas y que por tanto en la actualidad esta opción esta agotada. A este respecto, la evidencia empírica disponible para el caso de la economía mexicana, muestra resultados contradictorios. Por ejemplo, Murthy (1993) y Lin (1995) encuentran evidencia a favor de la ley de Wagner mientras que Nagarajan y Spears (1990) la rechazan. Puede sin embargo argumentarse, que estas divergencias en los resultados están, en alguna medida, asociados a diferencias en los métodos de estimación econométrica y, más importante aun que no se considera la posibilidad de cambios estructurales en esta relación. Esto es, la trayectoria del gasto públíco durante las últimas tres décadas ha mostrado ajustes estructurales importantes que pueden haber incidido en la forma en que se relaciona con el producto.

En este sentido, el principal objetivo de este artículo es analizar y evaluar la relación que se establece entre gasto público y producto en un contexto multivariado y atendiendo a la posible presencia de cambios estructurales en esta relación. Así, se realiza un análisis específico sobre la posible presencia de cambios estructurales en las series consideradas y sus relaciones en el contexto de raíces unitarias y cointegración. El artículo se divide en cuatro secciones. La primera es la introducción, la segunda se presenta la especificación del modelo y la metodología econométrica, en la tercera sección se muestra la evidencia empírica y finalmente se presentan las conclusiones.

\section{Hipótesis básicas y metodología econométrica}

La literatura económica tiene explicaciones alternativas sobre las relaciones de causalidad entre el gasto público y el producto. Por un lado, la hipótesis de 
Wagner postula que la actividad del gobierno, que normalmente se aproxima por la variable de gasto público, tiende a crecer conforme aumenta el desarrollo económico (medido por el ingreso per cápita). Ello es consecuencia del número creciente de demandas sociales y de intervención del estado en una economía desarrollada (Wagner y Webber, 1977). De este modo, la hipótesis de Wagner implica la presencia de una relación estable de largo plazo entre el gasto público y el ingreso per capita apoyada en una elasticidad superior a uno, y una causalidad unidireccional de producto hacia el gasto público (Chow, Cotsomitis y Kwans, 2002). Esto desde luego implica un aumento tendencial de la proporción de gasto público en el producto. Por el otro lado, el paradigma Keynesiano argumenta que el gasto público es una variable exógena y que por tanto su aumento genera un mayor crecimiento económico a través de dinamizar a la demanda agregada (Keynes, 1933). En este sentido, la causalidad es en la dirección contraria, del gasto público al producto.

El análisis de las relaciones de causalidad entre el producto per cápita y el gasto público y la posible presencia de cambios estructurales en estas relaciones puede realizarse en el marco de un modelo conocido como fundamental de capital (AK) (King y Levine, 1994). Este tipo de modelos, se basan en la especificación convencional de la ecuación de crecimiento que se define a partir de la siguiente función (Barro y Sala-i-Martin, 1995, p. 146):

$$
Y_{t}=F\left(A, K_{t}, L_{t}\right)
$$

Donde $K_{t}$ y $L_{t}$ representan capital y trabajo, respectivamente, y el factor $A$ se identifica como un índice de conocimientos o el nivel de tecnología, que a diferencia del modelo de crecimiento neoclásico tradicional no se asume como exógeno. Suponiendo entonces que la función de producción tiene la forma de una función Cobb-Douglas, el crecimiento económico tendencial puede entonces asociarse al comportamiento de la inversión o al acervo total de capital. ${ }^{1}$

La evidencia empírica de los modelos de crecimiento (Ross 2000, Feasel, Kim y Smith, 2001 y Bleaney y Nishiyama, 2002) utilizan, en muchos casos, como variable dependiente al producto per cápita ya que normalizan a la ecuación (1) por medio de la variable de empleo y asumen que la tasa de crecimiento de la fuerza laboral corresponde a la tasa de crecimiento de la población. ${ }^{2}$ De este modo, la hipótesis de Wagner y la hipótesis Keynesiana pueden ser anidadas en una ecuación que considera que el nivel de producto per capita a largo plazo esta determinado por la inversión y el gato público.

$$
y p_{t}=\beta_{0}+\beta_{1} i_{t}+\beta_{2} g p_{t}+u_{t}
$$

Donde las letras minúsculas representan el logaritmo natural de la serie, es decir $y p_{t}$ es el ingreso per cápita, $i_{t}$ la inversión total, y $g p_{t}$ el gasto público. Los parámetros $\beta_{1}$ y $\beta_{2}$ representan las elasticidades de inversión y de gasto

1 El capital y el trabajo a largo plazo crecen a una tasa constante, $K_{t}=K_{t-1}(1+g)$ y $L_{t}=L_{t-1}(1+n)$. Lo que se complementa con la siguiente identidad $K_{t}=K_{t-1}(1-$ $\gamma)+I_{t}$. Donde $I_{t}$ es la inversión y $\gamma$ es la tasa de depreciación del capital.

2 Vease las versiones aplicadas en Obstfeld y Rogoff, (1999), Ross (2000) y Favero, (2001). 
público respectivamente y $u_{t}$ representa el término de error. La estimación de la ecuación (2) requiere el uso de métodos econométricos que consideren el orden de integración de las series y el posible problema de regresión espuria (Granger y Newblod, 1974). El procedimiento de Johansen (1988) permite estimar las distintas relaciones de cointegración que pueden existir en un conjunto de variables, por medio de un modelo de vectores autorregresivos (VAR), en el caso de variables no estacionarias e identificar la posible presencia de cambio estructural en las series (Federici y Marconi, 2002). Asimismo, es posible analizar las condiciones de exogeneidad del modelo (Johansen, 1992) para conocer los casos donde el modelo econométrico estimado puede utilizarse para realizar inferencias estadísticas válidas, pronósticos y simulaciones de política económica (Ericsson e Irons, 1994).

La metodología econométrica consiste entonces, en primer lugar, en aplicar un conjunto de pruebas de raíces unitarias tales como la Dickey Fuller Aumentado (Dickey y Fuller, 1981) (ADF), la Phillips y Perron (1988) (PP) y la KPSS (Kwiatkowsky et al, 1992), a fin de identificar el orden de integración de las series utilizadas. Posteriormente, una vez establecido que las series son no estacionarias, se determinó la presencia de una relación de largo plazo entre el conjunto de variables consideradas incluyendo la posible existencia de cambio estructural en el vector de cointegracióna través del procedimiento de Johansen (1988).

El procedimiento de Johansen (1988) se basa en estimar un modelo VAR con $k$ rezagos, que permite describir el comportamiento estocástico de los datos y derivar entonces una prueba para analizar la existencia de relaciones de largo plazo entre las variables consideradas. De este modo, el VAR puede representarse como (Johansen, 1995):

$$
X_{t}=A_{1} X_{t-1}+\cdots+A_{k} X_{t-k}+u_{t}
$$

donde $X_{t}$ es un vector de $p \times 1$ dimensiones que incluye a todas las variables relevantes para el modelo y $u_{i}$ es $i . i . d$. $N\left(0, \sum\right)$. La ecuación (3) puede reparametrizarse como un modelo de corrección de errores (Johansen, 1988 y 1995) tal como:

$$
\Delta X_{t}=\sum_{i=1}^{k-1} \Gamma_{i} \Delta X_{t-i}+\Pi_{0} X_{t-1}+u_{t}
$$

Donde $\Gamma_{i}=-I+A_{1}+A_{2}+\cdots+A_{i}$, para $i=1,2, \cdots k-1$, y $\Pi_{0}=I-A_{1}-$ $\cdots-A_{k}$. En caso de que las variables incluidas inicialmente en el vector $X_{t}$ sean de orden de integración $I(1)$ entonces las series en $\Delta X_{t}$ son estacionarias, es decir $I(0)$, y por lo tanto el sistema sólo esta balanceado cuando $\Pi_{0} X_{t-1}$ es $I(0)$. Esta condición se deriva del rango de la matriz $\Pi_{0}$, es decir, del número de relaciones linealmente independientes y estacionarias. Así, que para probar relaciones de cointegración en el conjunto de variables consideradas se requiere determinar el rango $(r)$ de la matriz $\Pi_{0}$ a través de identificar el número de vectores característicos de $\Pi_{0}$ que son estadísticamente diferentes de cero.

Si el rango de $\Pi_{0}$ es completo $(r=p)$, ello implica que las variables en $X_{t}$ son estacionarias en niveles; en el caso en que la matriz $\Pi_{0}$ es de rango cero $(r=0)$, el modelo VAR debe ser especificado en primeras diferencias y no existen entonces vectores de cointegración; finalmente si la matriz $\Pi_{0}$ es de rango $r$, 
tal que $0<r<p$, existen $r$ combinaciones linealmente independientes que son estacionarias, que definen a $r$ vectores de cointegración. En este caso, la matriz $\Pi_{0}$ puede descomponerse en dos submatrices de orden $p \times r$, conocidas como $\Pi_{0}=\alpha \beta^{\prime}$, donde $\beta^{\prime}$ es una matriz compuesta por los vectores de cointegración y $\alpha$ la matriz de ponderaciones. Los coeficiontes de la matriz $\alpha$, también permiten identificar las variables exógenas débiles en el sistema (Johansen, 1995), mediante la significancia estadística de los coeficientes de la matriz.

La condición de exogeneidad débil (Ericson y Irons, 1994), de las distintas variables endógenas puede evaluarse considerando el caso donde la fila correspondiente de la matriz $\alpha$ es cero (Mosconi, 1998):

$$
H_{0}(i): \alpha=A_{i \varphi}, \quad i=1, \cdots, p_{y}
$$

Donde $A_{i}$ es una matriz identidad con dimensión $p_{y}$, cuya columna $i$, correspondiente a la variable endógena $x_{i}$, es suprimida, y $p_{y}=r$ rango de cointegración. El estadístico de la prueba de exogeneidad se distribuye como una $\chi^{2}$ con $r$ grados de libertad bajo la hipótesis nula $H_{0}$.

El método de Johansen (1988 y 1995) propone dos estadísticos para probar el número de vectores de cointegración. El estadístico de la traza (Traza) y el de la raíz característica máxima (L-max). Lo estadísticos se definen con base en la razón de máxima verosimilitud. Así el estadístico de la traza se representa como:

$$
-2 \ln Q=-T \sum_{i=r+1}^{p} \ln \left(1+\hat{\lambda}_{i}\right)
$$

Donde $\hat{\lambda}_{r+1}, \cdots, \hat{\lambda}_{p}$ son estimadas de los $p-r$ menores valores característicos. La hipótesis nula de la prueba asume que el número de vectores de cointegración es menor o igual que $r$, donde $r$ es $0,1,2, \cdots, r-p$. Alternativamente, el estadístico de la raíz característica máxima se define como:

$$
-2 \ln Q=-T \ln \left(1-\hat{\lambda}_{r+1}\right)
$$

En esta prueba, la hipótesis nula asume $r$ vectores de cointegración con respecto a la alternativa de $r+1$ vectores de cointegración. El uso de un modelo VAR que contenga un vector de cointegración y su respectivo vector de ponderaciones, permite resolver el problema de regresiones espurias y el sesgo en los estimadores y hace posible también analizar los supuestos de exogeneidad (Johansen, 1988 y 1995).

La presencia de posibles cambios estructurales en las relaciones entre las variables, se evalúan a través de dos pruebas definidas a partir del procedimiento de Johansen (Hansen-Johansen, 1993). Estas pruebas se construyen a partir de dos representaciones del modelo VAR. La representación Z o "modelo-Z", donde todos los parámetros del modelo (4) son estimados de manera recursiva, la segunda representación se denomina "modelo-R" en la cual los parámetros de corto plazo $\Gamma_{i}$ permanecen fijos para toda la muestra y únicamente los parámetros $\alpha$ y $\beta^{\prime}$ son re-estimados.

La primera prueba, denominada "prueba de Rango" examina la hipótesis nula de independencia en la muestra para el rango de cointegración del sistema. 
Esto es, con base en los residuales de la estimación recursiva, tanto en los modelos $\mathrm{R}$ y $\mathrm{Z}$, se obtienen las matrices de momentos para el procedimiento de Johansen, con ello se construye una secuencia de estadísticos de la traza, se rechaza la hipótesis nula de estabilidad si en alguna sub-muestra la prueba de la traza selecciona un rango distinto al estimado inicialmente para toda la muestra. En el caso de que los resultados en ambas especificaciones, modelo- $R$ y Modelo-Z, reporten resultados contradictorios la evidencia empírica sugiere aceptar el modelo-R (Hansen y Johansen, 1993).

La segunda prueba, asume como hipótesis nula la estabilidad del espacio de cointegración $S p\left(\beta^{*}\right)$, para un determinado rango de cointegración (Hansen y Johansen, 1993). La prueba utiliza la razón de máxima verosimilitud, que se obtiene de la función de verosimilitud de la estimación recursiva de cada sub-muestra y de la función de verosimilitud estimada para toda la muestra. El estadístico de la prueba se distribuye como una $\chi^{2}$ con $(p-r) r$ grados de libertad. En el caso en que la hipótesis nula no es rechazada se observa que el $S p\left(\beta^{*}\right)$ estimado para cada sub-muestra no es significativamente diferente al estimado en toda la muestra. El rechazo de la hipótesis nula indica que existe cambio estructural en el período considerado (Mosconi, 1998).

\section{La evidencia empírica}

La base de datos utilizada consiste en series anuales para el período 1970 al 2005. El producto per cápita $\left(Y P_{t}\right)$ es se define como el cociente del Producto Interno Bruto a precios de 1993 por el total de la población, la inversión total $\left(I_{t}\right)$ es aproximada por la formación bruta de capital total en millones de pesos a precios de 1993 y como variable de gasto público $\left(G P_{t}\right)$, corresponde al gasto programable del gobierno federal en millones de pesos a precios de 1993 .

El Cuadro 1, presenta los resultados de las pruebas de raíz unitaria de Dickey-Fuller Aumentada (1981), Phillips-Perron (1988) y la prueba KPSS (Kwiatkowsky et al., 1992). En el caso de las pruebas ADF y PPP, se aplicó el procedimiento "de lo general a lo específico" estimando en principio regresiones con constante y tendencia y verificando su significancia estadística. La prueba KPSS, utiliza como hipótesis nula el que la serie es estacionaria, a diferencia de las pruebas ADF y PP que tienen como hipótesis nula el que la serie incluye raíz unitaria (Maddala y Kim, 1998). La especificación de la prueba KPSS se realiza considerando que la serie es estacionaria alrededor de un término constante $\left(\eta_{\mu}\right)$; ó bien alrededor de una tendencia determinística $\left(\eta_{\tau}\right)$. Las letras minúsculas representan el logaritmo natural de las series.

Los resultados de las pruebas de raíces unitarias indican que el ingreso per cápita y la inversión total son series no estacionarias de orden de integración I(1). Ello sugiere además que el proceso de crecimiento de la economía mexicana sigue un patrón de fluctuaciones alrededor de una tendencia estocástica (Blanchard, 1997, Fisher, 1988 y Solow, 1997). En el caso del gasto público las pruebas ADF y PP indican que la serie puede ser estacionara alrededor de un término constante. Es importante mencionar que en el caso del gasto público, durante la década de los setentas el sector público adquirió un peso relevante en la actividad económica. ${ }^{3}$ Sin embargo, con la crisis de 1982 inicia una etapa de

3 Durante el periodo de 1971 a 1977, el gasto público creció a una tasa promedio anual 
estancamiento económico y de ajuste estructural. Entre las principales medidas, destaca la reducción del gasto público con el fin de reducir el déficit fiscal y los niveles de inflación. Estos cambios en la política económica han modificado de manera permanente la trayectoria de la serie que muestra un claro rompimiento en la tendencia en 1986 lo que incide en las pruebas de raíces unitarias y que por tanto hace importante analizar la presencia de una relación estable de largo plazo entre el gasto público y el producto per capita.

Cuadro 1. Pruebas de raíces unitarias.

\begin{tabular}{ccccccccc}
\hline Var. & \multicolumn{3}{c}{ ADF } & \multicolumn{3}{c}{ PP $(3)$} & \multicolumn{3}{c}{ KPSS $(6)$} \\
& $\mathrm{A}$ & $\mathrm{B}$ & $\mathrm{C}$ & $\mathrm{A}$ & $\mathrm{B}$ & $\mathrm{C}$ & $\eta_{\mu}$ & $\eta_{\tau}$ \\
\hline$y p_{t}$ & $-2.89(1)$ & $-2.06(0)$ & $2.24(0)$ & -2.51 & -2.03 & 1.97 & $\mathbf{0 . 5 5}$ & 0.11 \\
$\Delta y p_{t}$ & $\mathbf{- 4 . 5 7 ( 0 )}$ & $\mathbf{- 4 . 4 4 ( 0 )}$ & $-4.01(0)$ & -3.06 & $\mathbf{- 4 . 4 3}$ & $\mathbf{- 4 . 0 1}$ & 0.21 & 0.10 \\
$i_{t}$ & $\mathbf{- 4 . 3 6}(1)$ & $-0.93(0)$ & $2.16(0)$ & -3.22 & -0.87 & 2.54 & $\mathbf{0 . 6 1}$ & 0.08 \\
$\Delta i_{t}$ & $\mathbf{- 4 . 5 9}(1)$ & $\mathbf{- 4 . 6 6}(1)$ & $\mathbf{- 4 . 5 3}(0)$ & $\mathbf{- 4 . 3 0}$ & $\mathbf{- 4 . 9 4}$ & $\mathbf{- 4 . 4 5}$ & 0.11 & $\mathbf{0 . 1 8}$ \\
$g p_{t}$ & $\mathbf{- 4 . 4 0}(0)$ & $\mathbf{- 3 . 0 8}(0)$ & $2.41(0)$ & -2.48 & $\mathbf{- 3 . 1 5}$ & $\mathbf{- 4 . 3 7}$ & $\mathbf{0 . 4 5}$ & 0.13 \\
$\Delta g p_{t}$ & $\mathbf{- 5 . 2 7}(0)$ & $\mathbf{- 4 . 0 9}(0)$ & $\mathbf{- 4 . 3 2}(0)$ & $\mathbf{- 5 . 3 0}$ & $\mathbf{- 4 . 9 5}$ & $\mathbf{- 4 . 3 7}$ & 0.27 & $\mathbf{0 . 1 4}$ \\
\hline
\end{tabular}

Nota: Los número en negrilla representan rechazo de la hipótesis nula. El valor crítico es al $5 \%$ de significancia para la Dickey-Fuller y la Phillips Perron para muestras de hasta $\mathrm{T}=100$ son -3.45 incluye constante y tendencia (modelo A), -2.89 incluye constante (modelo B) y -1.95 sin constante ni tendencia (modelo C) (Maddala and Kim, 1998, p. 64). $\eta_{\mu}$ y $\eta_{\tau}$ es la prueba KPSS para la hipotesis nula de estacionariedad airededor de una constante y una tendencia lineal determinística respectivamente. El valor crítico de $5 \%$ para los dos pruebas son 0.463 y 0.146 respectivamente. (Kwiatkowski et al. 1992, p. 166). Periodo 1970-2004.

De este modo, los resultados de las pruebas de raíces unitarias sugieren que es necesario utilizar un procedimiento de estimación basado en las propiedades de cointegración. De esta manera se procedió a especificar un modelo VAR que cumpliera con los criterios de correcta especificación estadística (Cuadro A.1). En el Cuadro 2 se reportan los estadísticos de la traza y de la raíz característica máxima del procedimiento de Johansen (1988). Los resultados indican la presencia de al menos un vector de cointegración lo que sugiere la existencia de una relación de equilibrio entre el ingreso per cápita, la inversión total y el gasto público. Normalizando el vector de cointegración para el producto per cápita se obtiene:

$$
y p_{t}=5.08+0.133^{*} i_{t}+0.222^{*} g p_{t}
$$

Los resultados de la ecuación (8) muestran que el producto per cápita responde de manera positiva a cambios en los niveles de inversión y de gasto público. Sin embargo, ambos coeficientes son menores a uno lo que representa evidencia en contra de la hipótesis de Wagner. Esto es, la ley de Wagner, establece, que un incremento en el gasto público o un aumento en su participación en el gasto total, debe traducirse en un incremento en el ingreso per cápita en la misma proporción. Ello significaría que la elasticidad entre el ingreso per cápita y el gasto público debcría ser igual a uno (Courakis et al., 1993, Chow et al., 2002).

de 12.5 por ciento en términos reales, y en 1976 llegó a representar el 32 por ciento del PIB. 
Cuadro 2. Prueba de Cointegración de Johansen.

\begin{tabular}{ccccc}
\hline Ho & Traza & $95 \%$ & $\lambda-\max$ & $95 \%$ \\
\hline$r=0$ & $36.07^{*}$ & 34.91 & 14.94 & 22.0 \\
$r \leq 1$ & $36.55^{*}$ & 21.14 & 14.10 & 15.67 \\
$r \leq 2$ & 0.203 & 7.033 & 7.03 & 9.24 \\
\hline
\end{tabular}

Nota: $\left({ }^{*}\right)$ rechazo de la hipótesis nula a un nivel de significa de $5 \% ; \hat{\lambda}$ $\max =$ prueba de la raíz característica máxima (Johansen, 1995, tabla 15.1, p.214). Trace $=$ prueba de la Traza $($ Mackinnon et al., 1999, tabia II, p.571); $\mathrm{r}=$ número de vectores de cointegración rezagos $=3 . \quad \mathrm{El}$ modelo incluye constante. Periodo: 1970-2004

Sin embargo, la relación entre estas variables puede verse afectada por la presencia de cambio estructural. En efecto, durante el periodo de análisis la economía mexicana enfrento diversos cambios que han modificado su estructura productiva (Clavijo y Valdivieso, 2000). Durante la década de los setenta se registro una expansión del gasto público, principalmente apoyada por los ingresos de las exportaciones de petróleo. Con la caída de los precios internacionales del petróleo, así como las fuertes presiones económicas generadas por los desequilibrios fiscal y externo, la elevada inflación y la restricción del financiamiento del exterior llevaron a que las autoridades económicas del país, iniciaron un importante proceso de reformas estructurales en donde destaca la contracción del gasto público y la desincorporación, venta y liquidación de empresas estatales. Sin duda, estas medidas de política económica han tenido un impacto en la relación entre el ingreso per cápita y el gasto público que debe ser considerado.

Así, por medio de la estimación recursiva del procedimiento de Johansen es posible identificar la estabilidad de la relación de equilibrio entre el conjunto de variables. La prueba de estabilidad del rango de cointegración, se presenta en las Gráficas 1.a y 2.a, que corresponden a las especificaciones de los modelos $\mathrm{R}$ y $\mathrm{Z}$, respectivamente. Se observa que el estadístico de la traza reporta valores distintos en las sub-muestras indicando que el rango de cointegración no permanece estable en el tiempo. La prueba de estabilidad del espacio de cointegración, que se presenta en la Gráfica 3.a indica que en el caso del modelo-Z la razón de máxima verosimilitud rompe el valor crítico de 1 , en varios años a lo largo de la muestra rechazando la hipótesis de estabilidad del espacio de cointegración.

La inestabilidad del vector de cointegración en el tiempo hace necesario reespecificar el modelo VAR incluyendo los cambios en la tendencia de las series. En el ámbito de la econometría moderna, en años recientes, se ha generado una importante avance en la especificación de distintas pruebas de raíces unitarias en presencia de posibles cambios estructurales, identificando el momento de cambio estructural de manera endógena, toda vez que la elección del fecha de rompimiento en la tendencia de la serie no se puede realizarse sin considerar la información generada por los propios datos (Zivot y Andrews, 1992; Perron, 1997; Lumsdaine y Papell, 1997). 
Sin embargo, en el contexto multivariado, es dificil identificar correctamente las fechas de cambio estructural para el vector de cointegración. Por otra parte, es importante considerar que el periodo de análisis (1970-2004), comprende diversas etapas de la economía mexicana, donde se han presentado choques externos y medidas de política económica que han afectado la evolución de las variables especificadas en el modelo. Así, que la hipótesis de múltiples cambios estructurales en la serie es compatible con la presencia de fluctuaciones transitorias que desvían a la variable de su trayectoria de largo plazo. En este sentido, Bai y Perron (1998 y 2003), han desarrollado una metodología aplicada a las prueba de raíz unitaria, para identificar múltiples cambios estructurales en la serie, así como en un modelo lineal estimado por mínimos cuadrados ordinarios. ${ }^{4}$ La especificación del modelo de regresión lineal con " $m$ " cambios estructurales se define entonces como (Bai y Perron, 1998):

$$
y_{t}=x_{t}^{\prime} \beta+z_{t}^{\prime} \delta_{j}+u_{t} \quad i=T_{j-1}, \cdots, T_{j}
$$

Donde $y_{t}$ es la variable observada, $x_{t}$ contiene a las variables explicativas y $z_{t}$ es una matriz que contiene variables dummy que registran los cambios estructurales, $\beta$ y $\delta_{j}, j(j=1, \ldots, m+1)$ son los vectores de parámetros, $u_{t}$ el término de error y las fechas de cambio estructural están representadas por los puntos $\left(T_{1}, \cdots, T_{m}\right)$ que son desconocidos, y que son estimados junto con los parámetros con $T$ observaciones disponibles. En principio, la ecuación (9) se estima por el método de mínimos cuadrados ordinarios para $m$-particiones de la muestra $\left(T_{1} \cdots, T_{m}\right)$, el primer punto de cambio es identificado en donde se minimiza la suma de errores al cuadrado, que corresponde a una prueba de parámetros constantes. En ese punto la muestra es dividida en dos segmentos separados y en el segundo tramo de la muestra se sigue un procedimiento similar para estimar un nuevo punto de cambio estructural.

La especificación de la prueba para el caso de una serie individual considera como variable explicativa a un término constante que registra los cambios en la serie de tiempo, basado en una partición óptima (Bai y Perron, 1998). El Cuadro 3, reporta los resultados de la prueba Bai-Perron (1998 y 2003) para las series del ingreso per cápita y el gasto público. El modelo óptimo se elige con base en mínimo de la suma de errores al cuadrado y de acuerdo al Criterio Bayesiano de Información (BIC). En principio se consideró una partición de la muestra en cinco segmentos, asumiendo la presencia de cinco cambios estructurales. ${ }^{5}$ El estadístico BIC identifica tres posibles cambios estructurales para la serie del PIB per cópita para los años 1981, 1989 y 1996; por su parte la serie del gasto público señala que existen hasta cuatro cambios estructurales en las fechas de 1975, 1981, 1989 y 1998.

4 Si bien el modelo lineal estimado por mínimos cuadrados ordinarios, no corresponde al vector de cointegración estimado por el procedimiento de Johansen (1988), es una buena guía para determinar múltiples cambios estructurales.

5 La prueba se realizó. 
Cuadro 3. Prueba Bai-Perron de múltiples cambios estructurales.

\begin{tabular}{|c|c|c|c|c|c|}
\hline \multicolumn{3}{|c|}{ PIB per cápita $\left(y p_{t}\right)$} & \multicolumn{3}{|c|}{ Gasto público $\left(g_{t}\right)$} \\
\hline $\begin{array}{l}\text { Número } \\
\text { de } \\
\text { cambios }\end{array}$ & $\mathrm{BIC}$ & $\begin{array}{l}\text { Fechas } \\
\text { de } \\
\text { cambio }\end{array}$ & $\begin{array}{c}\text { Número } \\
\text { de } \\
\text { cambios }\end{array}$ & $\mathrm{BIC}$ & $\begin{array}{l}\text { Fechas } \\
\text { de } \\
\text { cambio }\end{array}$ \\
\hline 1 & -6.6862 & 1981 & 1 & -4.5391 & 1981 \\
\hline 2 & -6.8974 & 1981,1996 & 2 & -4.6566 & 1981,1998 \\
\hline 3 & -6.9733 & $1981,1989,1996$ & 3 & -4.7677 & $1975,1981,1998$ \\
\hline 4 & -6.7950 & $\begin{array}{l}1977,1982,1989 \\
1996,\end{array}$ & 4 & -4.8274 & $\begin{array}{l}1975,1981,1989, \\
1998\end{array}$ \\
\hline 5 & -6.5521 & $\begin{array}{l}1977,1982,1987 \\
1992,1997\end{array}$ & 5 & -4.6884 & $\begin{array}{l}1975,1981,1988 \\
1993,1998\end{array}$ \\
\hline
\end{tabular}

Los resultados de la prueba de Bai-Perron (1998 y 2003), sugieren diferentes fechas de cambio estructural por lo que fue necesario realizar una reespecifico del modelo VAR incluyendo variables dummys de pulso, para las distintas fechas señaladas de cambio estructural y mediante proceso de lo general a lo especifico se obtuvo un modelo final que incluye tres cambios estructurales en los años de 1975, 1981 y 1988. La incorporación de variables dummy en el procedimiento de Johansen (1988) modifica los valores críticos de las pruebas estadísticas (Mosconi, Johansen y Nilsen, 2000). La estimación de los nuevos valores críticos se utilizó el programa DisCo ${ }^{6}$ (Johansen y Nilsen, 1993). En el Cuadro 4, se presentan los resultados de la prueba de la traza con los nuevos valores críticos, indicando la presencia de dos vectores de cointegración.

Cuadro 4. Prueba de cointegración de Johansen suponiendo cambio estructural.

\begin{tabular}{ccc}
\hline Ho & Traza & $95 \%$ \\
\hline$r=0$ & $36.73^{*}$ & 33.74 \\
$r \leq 1$ & $16.29^{*}$ & 17.71 \\
$r \leq 2$ & 2.55 & 8.85 \\
\hline
\end{tabular}

Nota: $\left(^{*}\right.$ ) rechazo de la hipótesis nula al 5\% de significancia. Traze = prueba de la Traza. Valores críticos calculados por el DisCo con 20,000 simulaciones para una muestra de 100 observaciones. El modelo VAR(1), incluye constante, variables dummy en 1975,1981 y 1988. Periodo 1970-2004.

${ }^{6}$ El programa DisCo simula el límite de la distribución de la prueba del rango de cointegración asumiendo la presencia de cambio estructural en el componente determinístico del modelo VAR. 
El análisis de estabilidad del rango de cointegración con base en los modelos $\mathrm{Z}$ y $R$, que se presentan en las Gráficas 1 y 2 donde se observa que tanto para el modelo $\mathrm{R}$ como para el modelo $\mathrm{Z}$ el rango de cointegración es estable a lo largo del periodo. Esto es, el rango de cointegración $\mathrm{r}=0$ es rechazado, es decir en las submuestras reporta a valores menores a uno, en tanto que el rango de cointegración $\mathbf{r}=1$ no es rechazado, toda vez que en toda las submuestras reporta valores mayores a uno. Por su parte, la prueba de estabilidad en el espacio de cointegración $s p\left(\beta^{*}\right)$, la razón de máxima verosimilitud, tanto en la especificación del modelo-Z y del modelo- $\mathrm{R}$ (Gráfica 3 ), se observa que en las distintas submuestras se reportan valores menores a uno, indicando que no se rechaza la hipótesis nula de estabilidad en el espacio de cointegración. Estos resultados indican que existe una relación estable entre el producto per cápita, la inversión privada y el gasto público incluyendo un variables dummy en 1975, 1981 y 1988.

Gráfica 1. Prueba de estabilidad del rango de cointegración (Modelo R) Modelo con cambio estructural (Nivel de significancia 95\%).

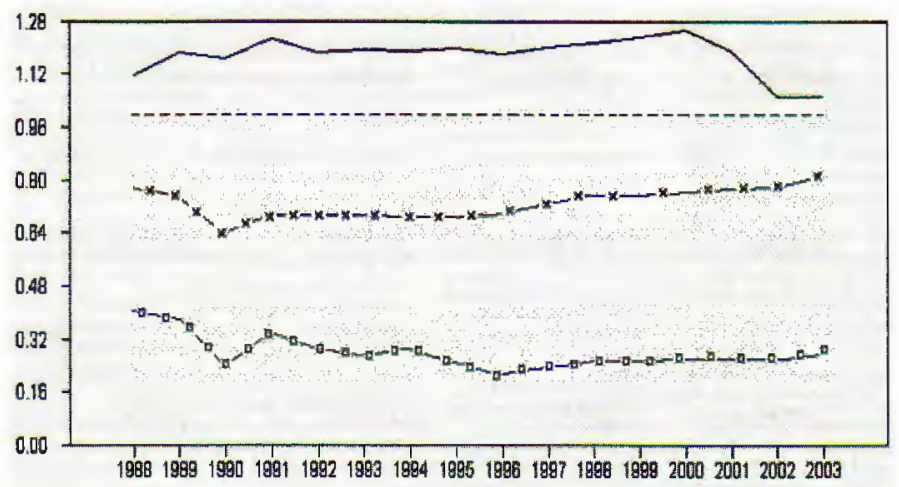

Gráfica 2. Prueba de estabilidad del rango de cointegración (Modelo Z)

Modelo con cambio estructural (Nivel de significancia 95\%).

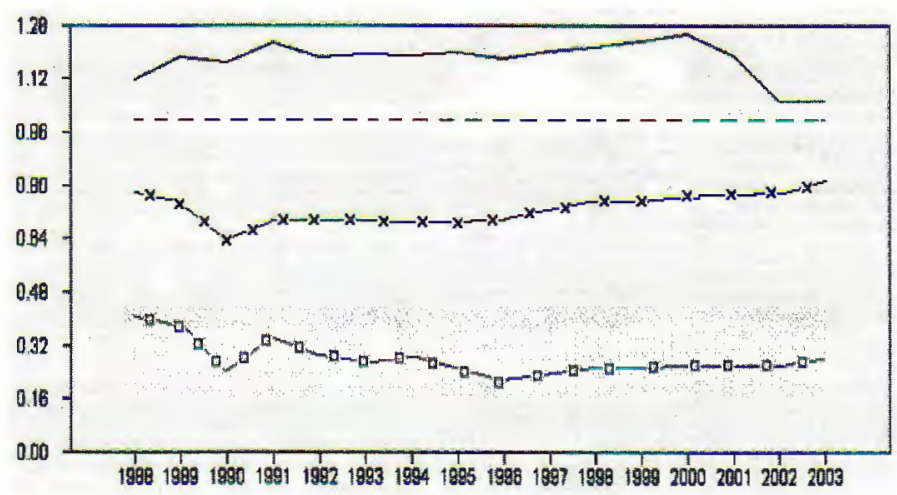


Gráfica 3. Prueba de estabilidad del espacio de cointegración (Modelo R y Z) Modelo con cambio estructural (Nivel de significancia 95\%).

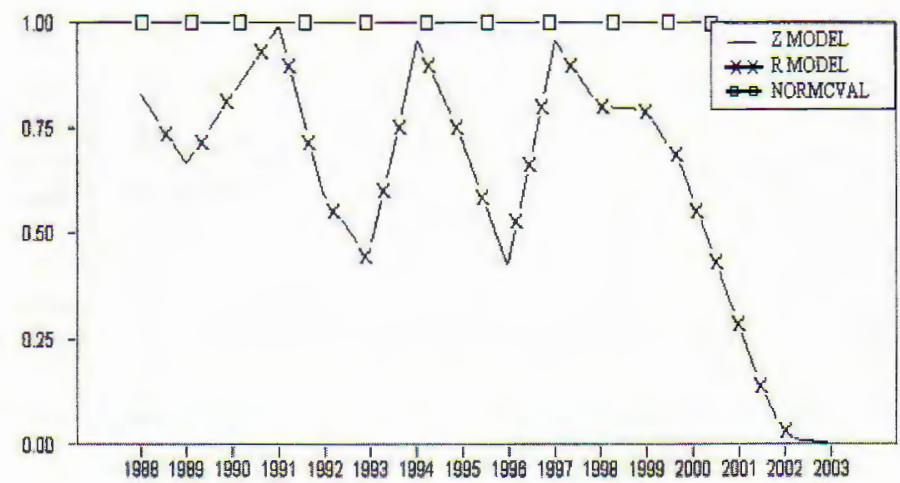

Normalizando al vector de cointegración como una ecuación de producto se observa que la inversión privada y el gasto público tienen una relación positiva con respecto al producto per cápita. Más aún destaca que el coeficiente de la relación entre el producto per cápita y el gasto público es menor que la unidad lo que es evidencia en, estricto sentido, contra de la hipótesis de Wagner. Este resultado corresponde a la reducción paulatina del gasto público asociado a los procesos de ajuste fiscal durante la década de los ochenta (Lustig, 1992).

$$
y_{t}=5.1+0.104^{*} i_{t}+0.256^{*} g_{t}
$$

Estos resultados son consistentes con la evidencia internacional tales como Chang (2002) y Lin (2002) y Chow, Cotsomits and Kwan (2002) donde se argumenta que existe una relación de cointegración entre ingreso y gasto público para el caso de Estados Unidos e Inglaterra. Por su parte, la evidencia para México es mixta. Por un lado, Murthy (1993) utilizando datos anuales de 1950 a 1980 encuentra cointegración ente el ingreso per cápita y la participación del gasto público en el producto mientras que Ashworth (1994) y Hayo (1994) no encuentran una relación estable entre gasto público y producto. En este sentido, los resultados obtenidos en este trabajo, permiten inferir las causas que explican los resultados anteriores asociadas al cambio en la muestra, al uso de un contexto multivariado y a la necesidad de incorporar, en al análisis, la posible presencia de cambios estructurales. Esto es, el análisis de la relación entre gasto público y producto debe realizarse en el contexto de un análisis multivariado que permite además, incorporar la presencia de cambios estructurales en las relaciones de largo plazo.

La representación del modelo VAR ordenado como producto per cápita, inversión y gasto público, en su forma de corrección de errores (VECM), permite además realizar las pruebas respectivas de exogeneidad débil (Ericsson y Irons, 1994). Las pruebas realizadas, sintetizadas en el Cuadro 5, indican que la inversión privada no rechaza la hipótesis de exogeneidad débil, indicando que esta variable pude ser utilizada en el largo plazo para influir en la trayectoria del producto per cápita. En cambio el gasto público, rechaza la hipótesis de exogeneidad débil reflejando que existe un fuerte proceso de retroalimentación 
entre el gasto público y el ingreso per cápita. En este caso, un cambio en el gasto público tendrá efectos en la trayectoria del ingreso per cápita, pero este resultado a su vez afectará la trayectoria del gasto público. De este modo, cualquier desviación en la relación de equilibrio sólo se ajusta de manera parcial en cada año. En este sentido, la hipótesis Keynesiana de utilizar el gasto público como una variable completamente exógena para inducir el crecimiento económico en el largo plazo no es aceptada por los datos. Esta evidencia es compatible con las investigaciones de Ansari, Gordon y Akuamoah (1997), quiénes argumentan en contra de la hipótesis Keynesiana para tres países de África mientras que es contraria a Holmes y Hutton (1990) que apoya el argumento Keynesiano para el caso de la India.

Cuadro 5. Pruebas de exogeneidad débil.

\begin{tabular}{cc}
\hline Null hypothesis Ho: $\alpha_{i}=0$ & \\
\hline$\alpha_{0}=0$ & $\chi^{2}(1)=3.437[0.064]^{*}$ \\
$\alpha 1=0$ & $\chi^{2}(1)=1.830[0.1761]$ \\
$\alpha 2=0$ & $\chi^{2}(1)=5.735[0.016]^{* *}$ \\
\hline
\end{tabular}

Nota: $\left(^{*}\right)$ y $\left(^{* *}\right)$ indican el rechazo de la hipótesis nula al nivel de 5 y 10 por ciento, respectivamente. $\alpha_{0}$ representa el PIB per cápita, $\alpha_{1}$ es la inversión privada y 2 representa el gasto público. Periodo 1970-2004.

Las pruebas de no causalidad en el sentido de Granger se reportan en el Cuadro 6. La prueba se especifico con las primeras diferencia de todas las variables, incluyendo los efectos contemporáneos y los errores del vector de cointegración y las estimaciones se realizaron con dos rezagos. Los resultados indican la presencia de una causalidad bi-direccional a corto plazo, entre el gasto público y el producto per cápita, así una expansión del gasto público genera un aumento en el ingreso per cápita que influye a su vez en el gasto público. Esto es cualquier desviación de la posición de equilibrio es corregida mediante un proceso dinámico entre ambas variables a lo largo del tiempo. Las pruebas de causalidad en los dos rezagos de cada variable no rechazan la hipótesis nula, sugiriendo que no existen efectos entre los rezagos de las variaciones del gasto y el ingreso per cápita. Sin embargo, la prueba $F$ sobre los impactos de los rezagos del gasto público sobre el producto per cápita rechaza la hipótesis nula a un nivel de significancia del $10 \%$, indicando un posible efecto del gasto público sobre el producto acorde con la hipótesis keynesiana. Asimismo, no se puede argumentar a favor de la presencia de una relación entre los cambios del ingreso per cápita y el impacto de valores pasados del gasto público atendiendo a las pruebas de no causalidad de Granger. En este sentido, los casos extremos de las hipótesis de Wagner y de Keynes no representan una buena aproximación al caso de la economía mexicana. Por otra parte, la inversión y el producto per cápita muestran un fuerte proceso bi-direccional mientras que no existe una relación con el gasto público. Ello refleja el hecho de que el crecimiento económico es el resultado de un complejo conjunto de factores. En todo caso, el crecimiento económico requiere, dada la causalidad simultánea, entre el producto per cápita y la inversión, la sincronía entre este conjunto de variables. 
Cuadro 6. Pruebas de no causalidad en el sentido de Granger.

\begin{tabular}{lcc}
\hline Prueba & Contemporáneo & Dos rezagos \\
\hline$\Delta g p_{t}$ causa $\Delta y p_{t}$ & $\mathrm{t}=6.46(0.00)^{* *}$ & $\mathrm{~F}=2.71(0.09)$ \\
$\Delta i_{t}$ causa $\Delta y p_{t}$ & $\mathrm{t}=6.90(0.00)^{* *}$ & $\mathrm{~F}=7.89(0.00)^{* *}$ \\
$\Delta y p_{t}$ causa $\Delta g_{t}$ & $\mathrm{t}=3.54(0.00)^{* *}$ & $\mathrm{~F}=2.15(0.14)$ \\
$\Delta y p_{t}$ causa $\Delta i_{t}$ & $\mathrm{t}=6.90(0.00)^{* *}$ & $\mathrm{~F}=2.77(0.08)$ \\
$\Delta i_{t}$ causa $\Delta g p_{t}$ & $\mathrm{t}=-1.04(0.30)$ & $\mathrm{F}=1.44(0.25)$ \\
$\Delta g p_{t}$ causa $\Delta i_{t}$ & $\mathrm{t}=-2.68(0.01)^{* *}$ & $\mathrm{~F}=0.51(0.60)$ \\
\hline
\end{tabular}

$(* *)(*)$ rechazo de la hipótesis nula al 1 y 5 por ciento de significancia respectivamente

\section{Conclusiones y comentarios generales}

La evidencia presentada en este artículo indica la presencia, en México, de una relación de largo plazo entre el ingreso per cápita, la inversión privada y el gasto público aunque con la presencia de cambios estructurales importantes, que reflejan el proceso de ajuste de la economía mexicana de las últimas dos décadas. Los valores de los coeficientes del vector de cointegración indican que existe una asociación positiva entre estas variables. Además, se observa que la relación entre producto per cápita y gasto público se identifica con un coeficiente menor que la unidad. Ello es evidencia en contra de la hipótesis de Wagner y refleja el proceso de ajuste fiscal de la década de los noventa. En este sentido, la especificación mutivariada utilizada que permite incluir la presencia de posibles cambios estructurales en la relación de largo plazo es una mejor opción para anidar las hipótesis de Wagner y la Keynesiana, en contraposición al uso de modelos bi-variados. De hecho, el uso de este marco de análisis permite englobar resultados previos tales como las dificultades para encontrar una relación estable de largo plazo.

Las pruebas de exogeneidad débil, indican que la inversión puede ser utilizada en el largo plazo para influir en la trayectoria del producto per cápita. Por el contrario, el gasto público rechaza la hipótesis de exogeneidad débil, indicando que existe un fuerte proceso de retroalimentación entre el gasto público y el ingreso per cápita. Este resultado es confirmado por las pruebas de no causalidad en el sentido de Granger donde se muestra que existe un fuerte proceso de causalidad bi-direccional entre la inversión y el producto per cápita. Sin embargo, en el caso del gasto público sólo se identificaron efectos contemporáneos respecto al ingreso per cápita.

En este caso, un cambio en el gasto público tendrá efectos en la trayectoria del ingreso per cápita, pero este resultado a su vez afectará la trayectoria del gasto público, sólo en el corto plazo y por lo tanto estos efectos, tenderán anularse en el tiempo. En este sentido, la hipótesis Keynesiana de utilizar el gasto público como una variable completamente exógena para inducir el crecimiento económico en el largo plazo no es aceptada por los datos. Sin embargo, la evidencia no muestra que la actividad económica pueda inducir un aumento del gasto público como plantea la hipótesis de Wagner. En este sentido, los casos extremos de la hipótesis de Keynes o Wagner no parecen tener validez empírica en el caso de la economía mexicana. 


\section{Apéndice}

Cuadro A.1. Pruebas de especificación del modelo VAR.

\begin{tabular}{cccc}
\hline Variable & LM (2) & ARCH $(1)$ & Normality \\
\hline$y_{t}$ & $F(2,25)=0.441[0.648]$ & $F(1,25)=0.00[0.991]$ & $\chi^{2}(2)=3.673[0.159]$ \\
$i_{t}$ & $F(2,25)=1.343[0.279]$ & $F(1,25)=0.919[0.347]$ & $\chi^{2}(2)=6.427[0.040]^{*}$ \\
$g_{t}$ & $F(2,25)=0.923[0.410]$ & $F(1,25)=0.189[0.659]$ & $\chi^{2}(2)=2.007[0.366]$ \\
\hline
\end{tabular}

Note. ${ }^{(*)}$ rechazo de la hipótesis nula a un nivel de significancia de $5 \%$. LM $=$ Prueba de autocorrelación, $\mathrm{ARCH}=$ Prueba de Heteroskedasticidad y J-B $=$ prueba de normalidad. Las letras en minúsculas representan las variables en logaritmo. Periodo 1970 - 2004.

Gráfica 1.a Prueba de estabilidad del rango de cointegración (Modelo R) Ecuación (8) (Nivel de significancia 95\%).

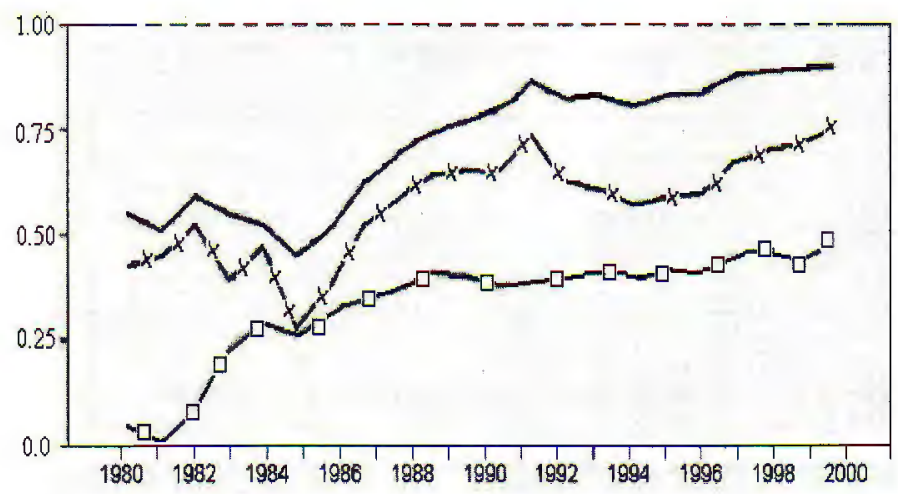

Gráfica 2.a Prueba de estabilidad del rango de cointegración (Modelo Z)

Ecuación (8) (Nivel de significancia 95\%).

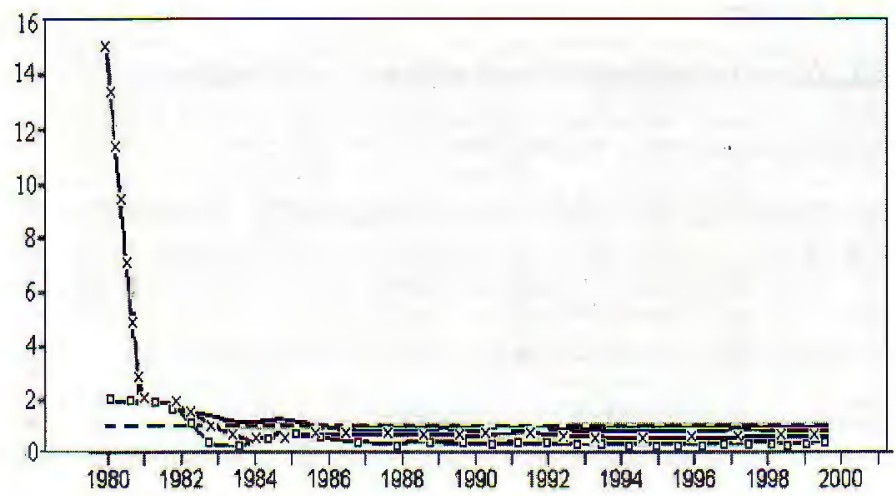


Gráfica 3.a Estabilidad del espacio de cointegración $\beta$ (Modelo R y Z) Ecuación (8) (Nivel de significancia 95\%).

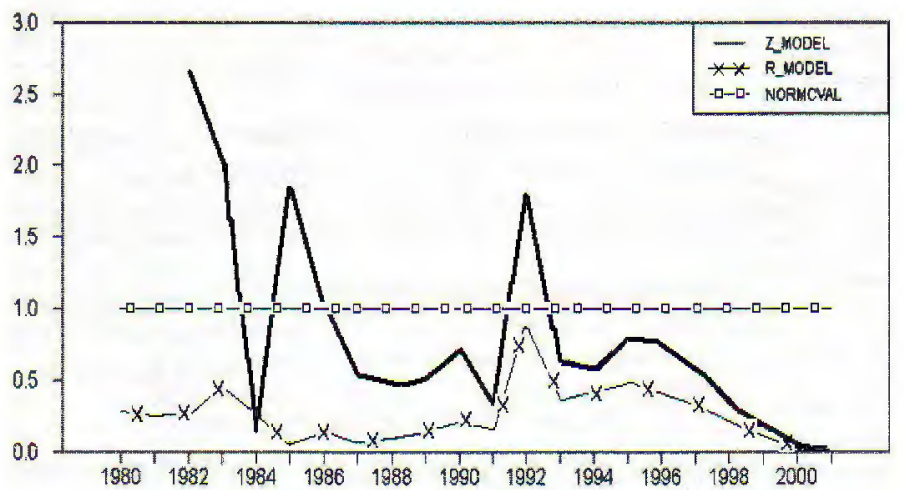

\section{Base de datos}

Todas las series son anuales de 1970 a 2004

$Y_{t}=$ Producto interno bruto per cápita (PIB per cápita) en millones de pesos de 1993, Instituto Nacional de Geografía e Informática (INEGI), Cuentas Nacionales.

$G_{t}=$ Gasto Público programable realizado en millones de pesos de 1993 Secretaría de Hacienda y Crédito Público (SHCP).

$I_{t}=$ Formación Fija de Capital del sector privado en millones de pesos de 1993, Instituto Nacional de Geografía e Informática (INEGI), Cuentas Nacionales.

\section{Bibliografía}

Ansari, M., D. Gordon and C. Akuamoah (1997) Keynes versus Wagner: Public Expenditure and National Income for three African Countries, Applied Economics, 29, pp. 543-550

Ashworth, J. (1994), Spurious in Mexico: a comment on Wagner's law, Public Finance / Finances Publiques, 49(2), pp. 282-286.

Bai, J. and P. Perron (1998). Estimating and Testing Linear Models with Multiple Structural Changes, Econometrica, 66, pp. 47-78.

Barro, T.J and X. Sala-I-Martin (1995). Economic Growth, New York: McGraw Hill.

Blanchard, O. (1997), Is there a core of usable macroeconomics?, American Economic Review, papers and proceedings, mayo, pp. 244-246.

Bleaney, M. and A. Nishiyama (2002), Explaining Growth: a contest between models, Journal of Economic Growth, 7, 43-56.

Chang, T. (2002). An econometric Test of Wagners law for six Countries based on Cointegration and Error-Correction Modeling Techniques, Applied Economics, 34, pp. 1157-1169

Chow, Y., J. Cotsomitis, and A. Kwan, (2002). Multivariate Cointegration and Causality Tests of Wagners Hypothesis: Evidence form the UK, Applied Economics, 34(13=, September, pp. 1671-1677. 
Clavijo, F. y S. Valdivieso (2000). La política industrial de México, 1988-1994, Lecturas del trimestre económico, FCE, No. 80.

Courakis, A., F. Moura-Roque, and G. Tridimas, (1993). Public Expenditure Growth in Grecce and Prtugal: Wagner's law and beyond, Applied Economics, 25(1), pp. 125 134

Dickey, D. and W. Fuller (1981). Likelihood Ratio Statistics for Autoregressive Time Series with a Unit Root, Econometrica, 49, pp.1057-1072

Ericsson, N. and J Irons, (1994). Testing Exogeneity, Oxford University Press

Favero, C. (2001). Applied Macroeconomics, Oxford University Feasel, E., Y. Kim y S. C. Smith (2001). Investment, Exports, and Output in South Korea: a VAR Approach to Growth Economics, Review of Development Economics, 5(3), pp. 421-432.

Federci, D. and D. Marconi (2002). On Exports and Economic Growth: the Case of Italy, The Journal of international trade and Economic Development, 11(3) pp. 323-340.

Fischer, S. (1988). Recent Developments in Macroeconomics, Economic Journal, No 98, junio, pp. 294-339.

Granger, C. and P. Newbold (1974). Spurious Regressions in Econometrics, Journal of Econometrics, 53, pp. 211-244.

Hansen, H. and S. Johansen (1993). Recursive Estimation in Cointegrated VAR Models, University of Copenhagen, Institute of Mathematical Statistics, pre-print 93-1.

Hayo, B. (1994). No further evidence of Wagners Law for Mexico, Public Finance / Finances Publiques, Vol. 49(2), pp. 287-294

Holmes, J. and P. Hutton (1990. On the Causal Relationship between Government Expenditure and National Income, The Review of Economics and Statistics, 72, pp. 231-254

Johansen, S. (1995). Likelihood-based Inference in Cointegrated Vector Autoregressive Models, Oxford University Press

Johansen, S. (1992). Testing weak Exogeneity and the Order of Cointegration in UK Money Demand Data, Journal of Policy Modeling, 14(3), pp. 313-334.

Johansen, S. (1988). Statistical Analysis of Co-integrating Vector, Journal of Economics Dynamics and Control, 12, pp. 231-254.

Johansen, S. y B Nielsen (1993). Asymptotics for Cointegration Ranks Test in the Presence of Intervention Dummies, Manual for the Simulation Program DisCo, http://www.nuff.ox.ac.uk/users/nielsen/disco.html.

Keynes, J. (1936). The General Theory of Employment, Interest and Money, London: Macmillan

King R. and R. Levine (1994). Capital Fundamentalism Economic Development, and Economic Growth, Carnegie-Rochester Conference Series on Public Policy

Kwiatkowsky, D., Phillips, P., P. Schmidt and Y. Shin (1992). Testing the Null Hypothesis of Stationary against the Alternative of a Unit Root, Journal of Econometrics, 54, pp. 159-178

Lin, C, (1995). More Evidence on Wagners Law for Mexico, Public Finance / Finances Publiques, 50(2), pp. 267-277

Lin, C., (2002). On the Level of Persistence in Government Size: Time Series Evidence and Implications for the US, Applied Economics, 34(8), pp. 999-1005

Lumsdaine, R. and D. Papell (1997). Multiple Trend Breaks and the Unit-Root Hypothesis, The Review of Economics and Statistics, 79, May.

Lustig, N. (1992). México hacia la reconstrucción de una economía, Fondo de Cultura Económica, El Colegio de México.

Maddala, G. and I. Kim (1998). Unit Roots, Cointegration and Structural Change, Cambridge University Press

Mackinnon J., A. Haug and L. Michelis (1999), Numerical Distributions of Likelihood Ratio Tests for Cointegration, Journal of Applied Econometrics, 14, pp. 563-577

Mosconi, R. (1998). MALCOLM. Maximum Likelihood Cointegration Analysis of Linear Models. The Theory and Practice of Cointegration Analysis in RATS. Libreria Editrice Cafoscarina. Primera edición. Diciembre 1998. 
Mosconi R., S. Johansen and B. Nielsen, (2000). Cointegration Analysis in the Presence of Structural Breaks in the Deterministic Trend, Econometrics Joumal, Royal Economic Society, 3(2), pp. 216-249.

Murthy, N. (1993). Further Evidence of Wagners Law for Mexico: An Application of Cointegration Analysis, Public Finance / Finances Publiques, 48(1), pp. 92-96

Nagarajan, P, and A. Spears, (1990). An Econometric Test of Wagners Law for Mexico: A Reexamination, Public Finance / Finances Publiques, 45(1), pp. 165-168

Obstfeld, M. and K. Rogoff (1999). Foundations of International Macroeconomics, MIT Press.

Perron, P.(1997). Further Evidence on Breaking Trend Functions in Macroeconomic Variables, Journal of Econometrics, 80.

Phillips, P and P. Perron (1988). Testing for Unit Roots in Time Series Regression, Biometrika, 75 , pp. 335-346

Ross J., (2000). Development Theory \& the Economics of Growth, The University of Michigan Press.

Solow, R. (1997), Is there a Core of Usable Macroeconomics we Should all Believe in?, American Economic Review, papers and proceedings, mayo, pp. 230-232.

Wagner, A. (1890). Finanzwissenschaft, Leipzing

Wagner, R. and W. Weber, (1977). Wagner's law, Fiscal Institutions, and the Growth of Government, National Tax Journal, 30, pp. 59-69

Zivot and Andrews (1992). Further Evidence on the Great Crash, the Oil Price Shock, and the Unit Root Hypothesis, Journal of Business and Economic Statistics, 10(3). 